\title{
Zur Prognose von Erschütterungen aus Bahntunneln
}

\section{Wellenzahlintegrale und Finite-Element-Randelement- Berechnungen}

\author{
Dr.-Ing. Lutz Auersch, Bundesanstalt für Materialforschung und - \\ prüfung, Berlin
}

\section{Kurzfassung}

Zur Erschütterungsausbreitung an oberirdischen Bahnlinien gibt es gute Übereinstimmungen zwischen Messungen und der Theorie geschichteter Böden. Bei der Interpretation der Ergebnisse spielt die Rayleigh-Welle eine große Rolle. Je nach Frequenz und Wellenlänge hat die Rayleigh-Welle eine bestimmte Eindringtiefe und erreicht damit mehr oder weniger steife Bodenschichten. Damit bekommt man eine frequenzabhängige Bodensteifigkeit für die Erschütterungsprognose. Für die Wellenausbreitung in der Tiefe statt an der Bodenoberfläche müssen eigene Gesetzmäßigkeiten gefunden werden. Es werden die Punktlastlösungen im Frequenz-Wellenzahlbereich und durch Integration über die Wellenzahlen berechnet. Man erhält die Wellenfelder, die Terzspektren für verschiedene Entfernungen und Frequenzen. Es wird die Tiefenlage und das Bodenmodell (homogen, geschichtet und kontinuierlich zunehmende Steifigkeit) variiert. Die Rayleigh-Welle verliert ihre Bedeutung und stattdessen kann die Vollraumlösung zur Interpretation und Prognose verwendet werden. Es werden die Halbraumlösung mit und ohne Rayleigh-Welle und die Vollraumlösung in der Tiefe diskutiert und verglichen. Neben der Wellenausbreitung (der Transmission) werden auch Effekte der Erschütterungsanregung (der Emission) und der Übertragung in Gebäude (der Immission) mit Hilfe der Finite-Element-Randelement-Methode berechnet. Die Verteilung der dynamischen Achslast durch die Tunnelsohle ergibt eine Minderung gegenüber der Punktlastanregung. Bei der Immission hat man keine Freifeldanregung wie an der Bodenoberfläche. Man muss entweder neben der Wellenamplitude (Verschiebung oder Schwinggeschwindigkeit) in der Tiefe auch die Spannung der ankommenden Welle berücksichtigen, oder man muss die Freifeldamplituden nach Bodenaushub berechnen. Die Rechenergebnisse deuten darauf hin, dass man als Freifeldanregung die zweifache Vollraumlösung ansetzen kann.

\section{Autorenkontakt: lutz.auersch-saworski@bam.de}




\section{Einleitung}

Die BAM hat eine gut ausgearbeitetes Prognoseverfahren für Erschütterungen aus oberirdischem Schienenverkehr [1]. Es fehlen noch Ergänzungen für Erschütterungen aus Bahntunneln (Bild 1). Diese Ergänzungen zur Erschütterungsausbreitung in der Tiefe, zur Minderungswirkung durch den Bahntunnel und zur Erschütterungsimmission in der Tiefe werden hier vorgestellt. Der Tunnel und die Gebäudefundamente werden mit der kombinierten Finite-Element-Randelementmethode berechnet. Die Wellenausbreitung wird mit den Punktlastlösungen des Halb- oder Vollraumes berechnet.

Bild 1: Erschütterungsausbreitung von Bahntunneln in tiefe Gebäudegründungen

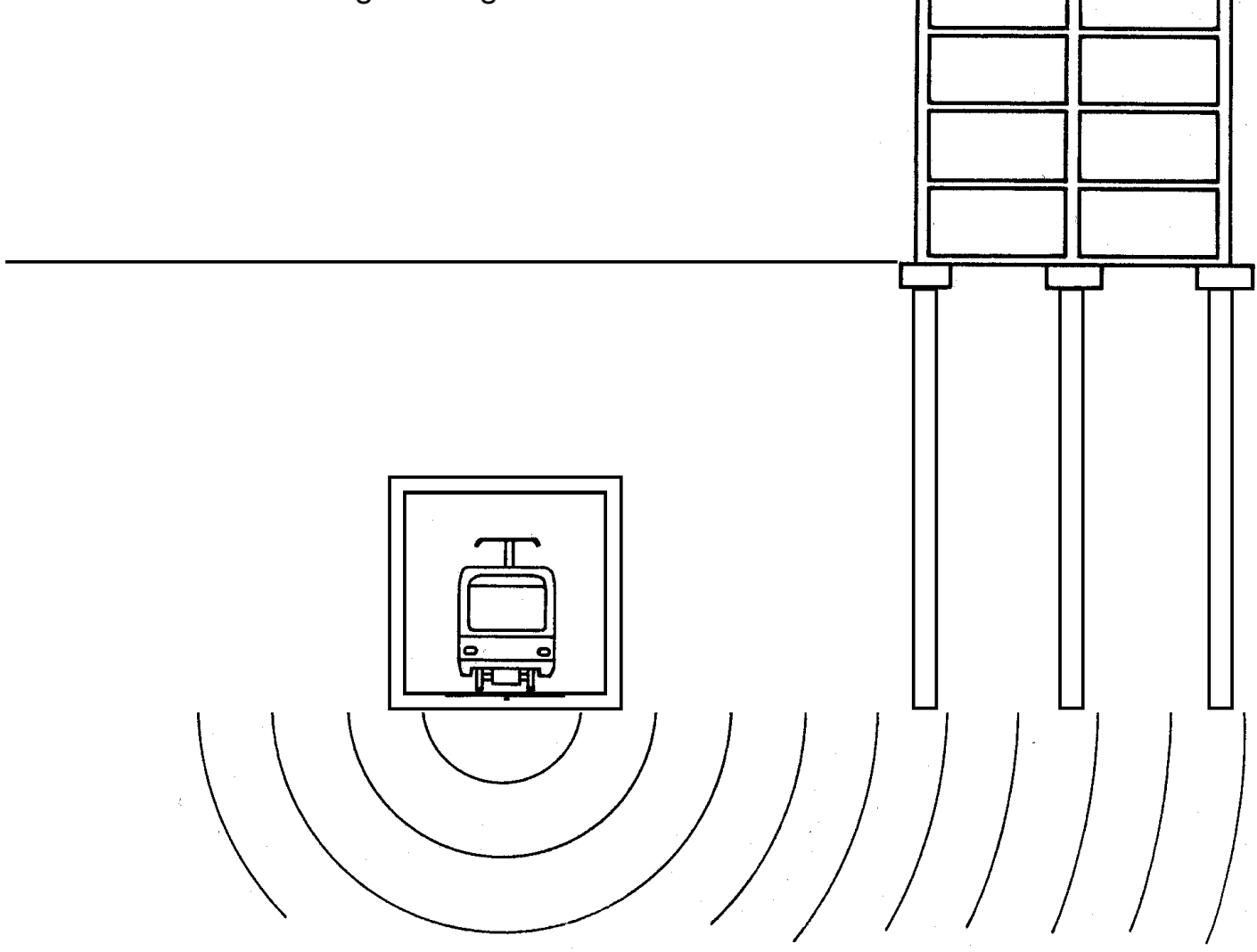

\section{Methoden}

\subsection{Punktlastlösungen des geschichteten Halbraums und des homogenen Vollraums}

Für die Wellenausbreitung im geschichteten Halbraum wird die Punktlastlösung mit folgendem Verfahren berechnet. Zu jeder Bodenschicht wird eine 4x4 komplexe symmetrische Steifigkeitsmatrix im Frequenz-Wellenzahlbereich berechnet [2]. Alle Schichtmatrizen werden zu Zur Prognose von Erschütterungen aus Bahntunneln AUERSCH

2. Wiener Dynamik Tage 2021 
einer globalen Steifigkeitsmatrix des Bodens zusammengefasst und zu einer globalen Nachgiebigkeitsmatrix $\mathbf{N}$ invertiert. Die interessierenden Elemente der Nachgiebigkeitsmatrix werden über die Wellenzahl $k$ integriert [3], zum Beispiel für die vertikale Komponente (mit der Besselfunktion $\mathrm{J}_{0}$ )

$$
C_{z z}\left(r, z_{1}, z_{2}\right)=\frac{1}{2 \pi} \int_{0}^{\infty} N_{z z}\left(k, z_{1}, z_{2}\right) \mathrm{J}_{0}(k r) k \mathrm{~d} k
$$

für eine Anregung in der Tiefe $z_{1}$ und einer Antwort im Abstand $r$ und in der Tiefe $z_{2}$.

Für den unendlichen Vollraum gibt es keine Tiefenparameter und die Lösung lässt sich explizit angeben als

$$
C_{z Z}(r)=\frac{1}{4 \pi G r}\left\{\left(1-\frac{1}{r^{* 2}}-i \frac{1}{r^{*}}\right) e^{-i r^{*}}+\left(\frac{1}{r^{* 2}}-i \frac{\beta}{r^{*}}\right) e^{-i \beta r^{*}}\right\}
$$

wobei $r^{*}=r \omega / v_{S}$ und $\beta=v_{S} / v_{P}$ und $G$ der Schubmodul ist. Diese Transversalkomponente kann für die horizontale Ausbreitung von vertikalen Schwingungen verwendet werden. Es existieren noch eine weitere Verschiebungskomponente und drei Spannungskomponenten, die ähnlich aufgebaut sind und die für die Randelementmethode eingesetzt werden [4].

\subsection{Die Randelementmethode}

Für die Randelementmethode wird die Kontaktfläche Boden-Struktur durch Punkte und zugehörige Flächen diskretisiert. Die Nachgiebigkeitsmatrix $\mathbf{C}$ des Bodens wird von einem Punkt zu einem anderen Punkt unmittelbar mit der Punktlastlösung aufgebaut. Für die Verschiebung des angeregten Punktes wird die Punktlastlösung über die zugehörige Fläche gemittelt, um eine endliche Nachgiebigkeit zu erhalten. Die voll besetzte Nachgiebigkeitsmatrix C wird zur dynamischen Steifigkeitsmatrix des Bodens $\mathbf{K}_{\mathrm{B}}$ invertiert.

$$
\mathbf{K}_{\mathrm{B}}(\omega)=\mathbf{C}^{-1}(\omega) \text {. }
$$

Bei der Vollraummethode muss auch eine Kraftmatrix $\mathbf{P}$ berechnet werden und die dynamische Steifigkeitsmatrix lautet

$$
\mathbf{K}_{\mathrm{B}}(\omega)=\mathbf{P}(\omega) \mathbf{C}^{-1}(\omega) \text {. }
$$

\subsection{Die gekoppelte Finite-Element-Randelement-Methode}

Eine Struktur (der Tunnel oder die Gebäudegründung) wird durch ein Finite-Element-Modell abgebildet, dessen Steifigkeitsmatrix $\mathbf{K}_{0}$ und Massenmatrix $\mathbf{M}$ ergeben eine dynamische Steifigkeitsmatrix $\mathbf{K}_{\mathrm{T}}(\omega)$

Zur Prognose von Erschütterungen aus Bahntunneln AUERSCH

2. Wiener Dynamik Tage 2021 


$$
\mathbf{K}_{\mathrm{T}}(\omega)=\mathbf{K}_{0}-\omega^{2} \mathbf{M}
$$

Die dynamischen Steifigkeitsmatrizen des Bodens und der Struktur werden addiert und ergeben die dynamischen Steifigkeitsmatrix $\mathbf{K}_{\mathrm{TB}}(\omega)$ des gekoppelten (Tunnel-Boden-) Systems

$$
\mathbf{K}_{\mathrm{TB}}(\omega)=\mathbf{K}_{\mathrm{T}}(\omega)+\mathbf{K}_{\mathrm{B}}(\omega)
$$

Die Antwort auf eine äußere Belastung $\mathbf{F}(\omega)$ erhält man dann als

$$
\mathbf{u}(\omega)=\mathbf{K}_{\mathrm{TB}^{-1}}(\omega) \mathbf{F}(\omega)
$$

die Verschiebungen $\mathbf{u}(\omega)$ des Struktur-Boden-Systems.

\section{Wellenausbreitung in der Tiefe}

Die Wellenausbreitung an der Bodenoberfläche ist weitgehend bekannt und mit den Eigenschaften der Rayleighwelle erklärt [3]. Aber bei Erschütterungen, die zum Beispiel von einem Bahntunnel ausgehen und auf eine Tiefgründung/ein Kellergeschoss eines Gebäudes treffen, sollen hier die Gesetzmäßigkeiten mithilfe der Punktlastlösungen ermittelt werden.

Für den homogenen Boden sind in Bild 2 die Wellenfelder (die Schwinggeschwindigkeiten in den Entfernungen $r=4,8,16,32,64 \mathrm{~m}$ und für Frequenzen zwischen 4 und $100 \mathrm{~Hz}$ ) bei vier verschiedenen Tiefenlagen der vertikalen Quelle und des Empfängers dargestellt. Die Schwinggeschwindigkeits- (Admittanz) Spektren nehmen mit der Frequenz zu. Bei größeren Entfernungen und höheren Frequenzen ergibt die Materialdämpfung (hier $D=2,5 \%$ ) einen horizontalen bis abnehmenden Frequenzverlauf. Mit zunehmender Tiefenlage gibt es eine zusätzliche Abminderung, die nur bei den höheren Frequenzen auftritt. Sie beginnt in etwa dort, wo ein typisches Minimum auftritt, in $4 \mathrm{~m}$ Tiefe bei $32 \mathrm{~Hz}$, in $8 \mathrm{~m}$ Tiefe bei $16 \mathrm{~Hz}$ und in $12 \mathrm{~m}$ bei $8 \mathrm{~Hz}$. Das heißt je tiefer die Anregung ist, desto tieffrequenter beginnt die Minderung. Oberhalb dieser Frequenz verhält sich der Boden wie ein elastischer Vollraum (Bild 2e). Bei den tiefen Frequenzen hat man die Amplituden des elastischen Halbraumes (Bild 2a). Bild $2 f$ zeigt den Unterschied zwischen der Halbraum- und der Vollraumlösung. Die Vollraumlösung ist maximal halb so groß wie die Halbraumlösung. Bei hohen Frequenzen oder großen Entfernungen enthält die Halbraumlösung den Rayleighwellenanteil, so dass der Unterschied größer wird. Betrachtet man zusätzlich die doppelte Vollraumlösung, dann stimmt diese mit der Halbraumlösung bis zum hochfrequenten Auftreten der Rayleighwelle überein.

Im nächsten Beispiel wird eine Bodenschichtung betrachtet, bei der eine weiche Schicht mit $v_{s}=150 \mathrm{~m} / \mathrm{s}$ und einer Dicke von $8 \mathrm{~m}$ über einem steiferen Boden mit mit $v_{s}=300 \mathrm{~m} / \mathrm{s}$ liegt.

Zur Prognose von Erschütterungen aus Bahntunneln AUERSCH

2. Wiener Dynamik Tage 2021 
a)

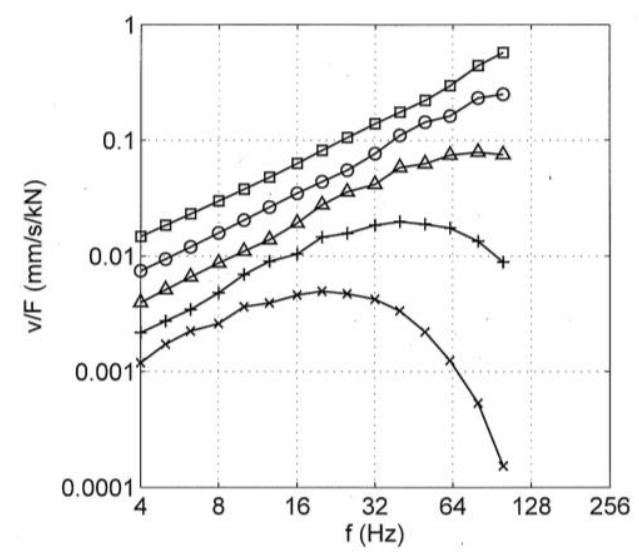

c)

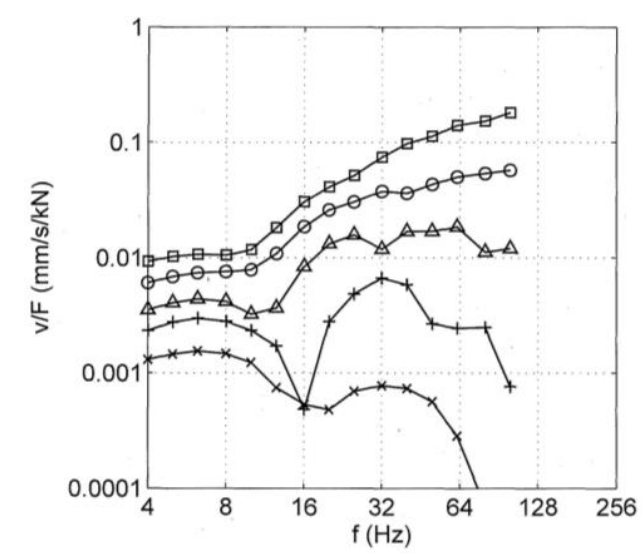

e)

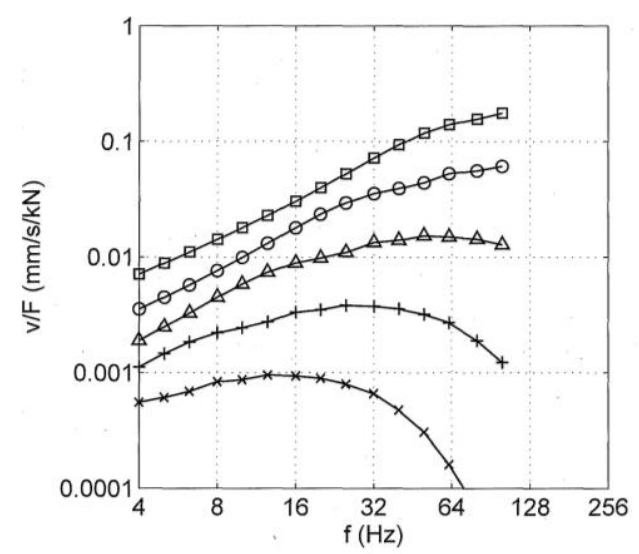

b)

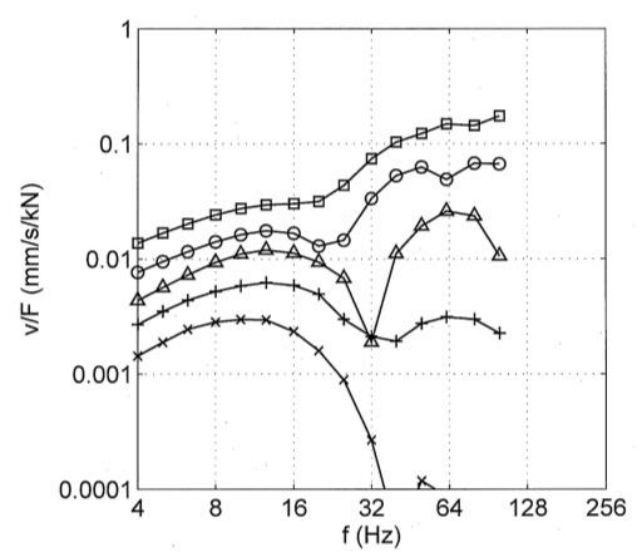

d)

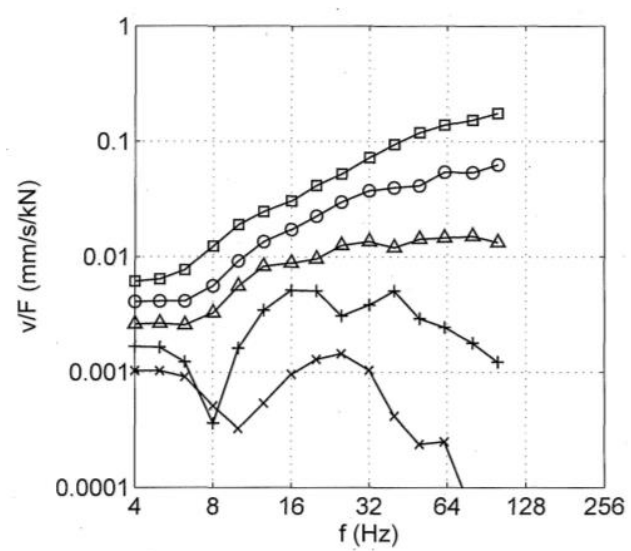

f)

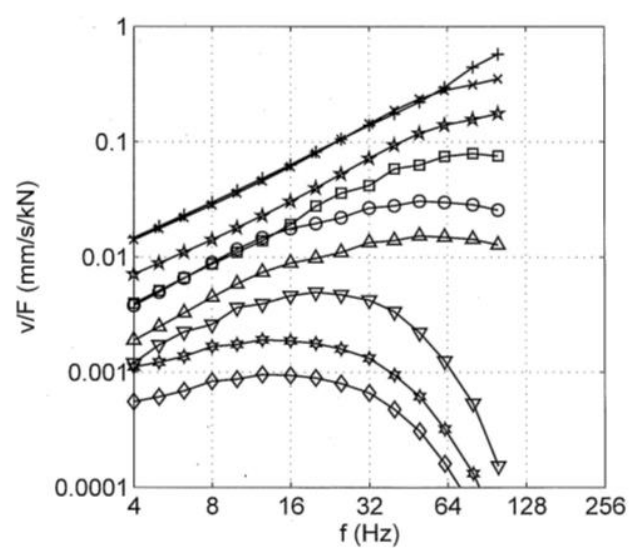

Bild 2: Das Wellenfeld eines homogenen Halbraums mit $v_{S}=150 \mathrm{~m} / \mathrm{s}$, Übertragungsfunktionen zwischen der Kraft und den Schwinggeschwindigkeiten im Abstand von $r=\square 4 \mathrm{~m}, \bigcirc 8 \mathrm{~m}$, $\triangle 16 \mathrm{~m},+32 \mathrm{~m}, \times 64 \mathrm{~m}$, a) an der Oberfläche, b) in $4 \mathrm{~m}$ Tiefe, c) in $8 \mathrm{~m}$ Tiefe, d) in $12 \mathrm{~m}$ Tiefe, e) des Vollraums mit $v_{s}=150 \mathrm{~m} / \mathrm{s}$, f) Vergleich Halbraum, $2 \times$ Vollraum, Vollraum bei $r=$ $+, \times, \star 4 \mathrm{~m}, \square, \bigcirc, \triangle 16 \mathrm{~m}, \nabla, *, \diamond 64 \mathrm{~m}$

An der Bodenoberfläche ergibt sich das typische Wellenausbreitungsbild einer Bodenschichtung (Bild 3a). Die hohen Frequenzen zeigen das Verhalten des weichen homogenen Halb-

Zur Prognose von Erschütterungen aus Bahntunneln AUERSCH

2. Wiener Dynamik Tage 2021 
a)

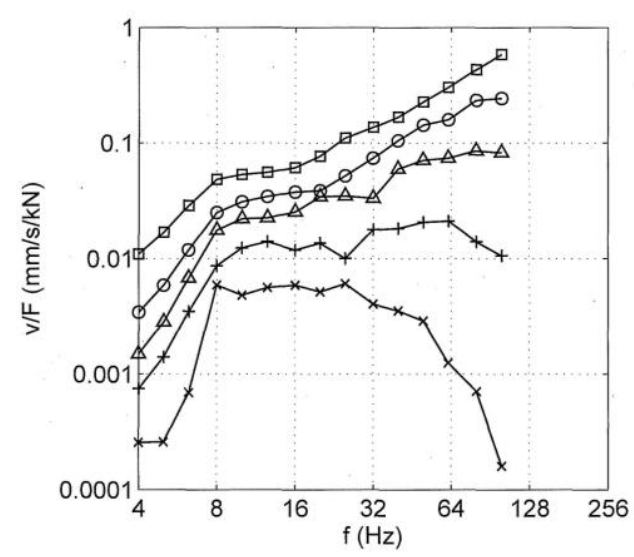

c)

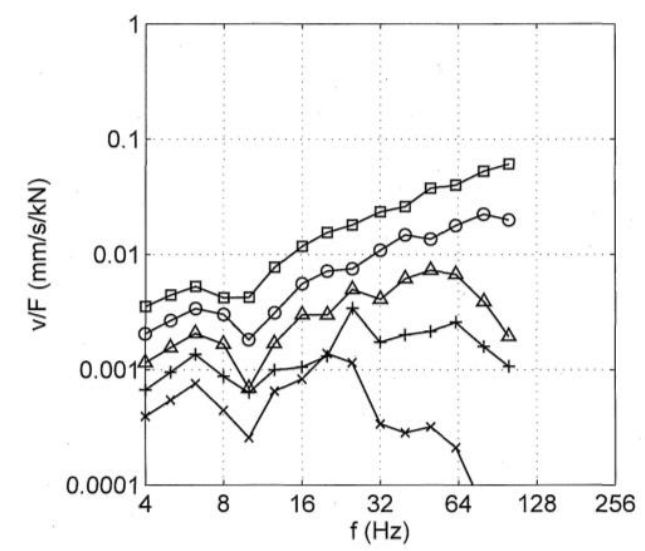

e)

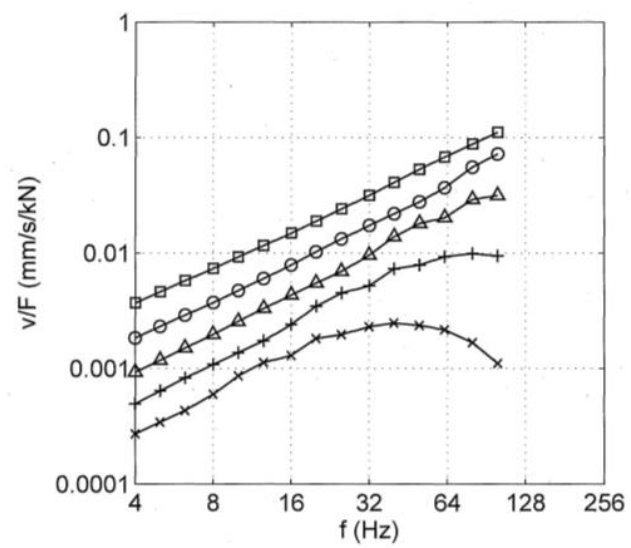

b)

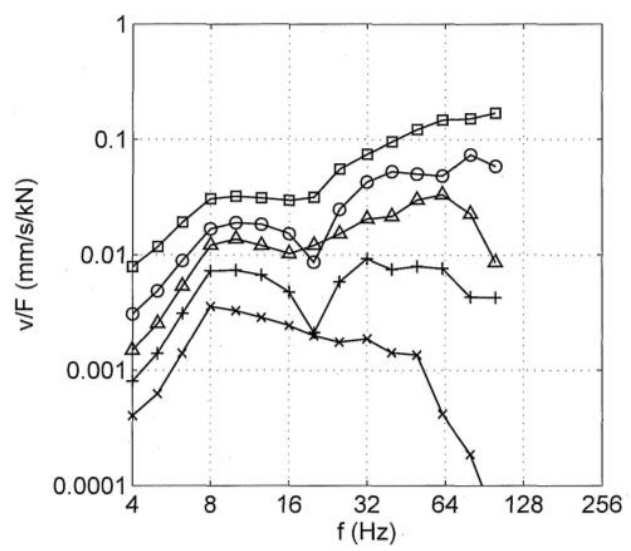

d)

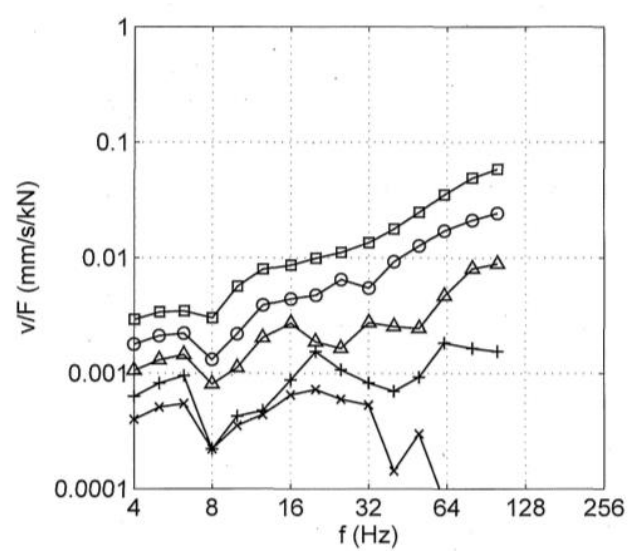

f)

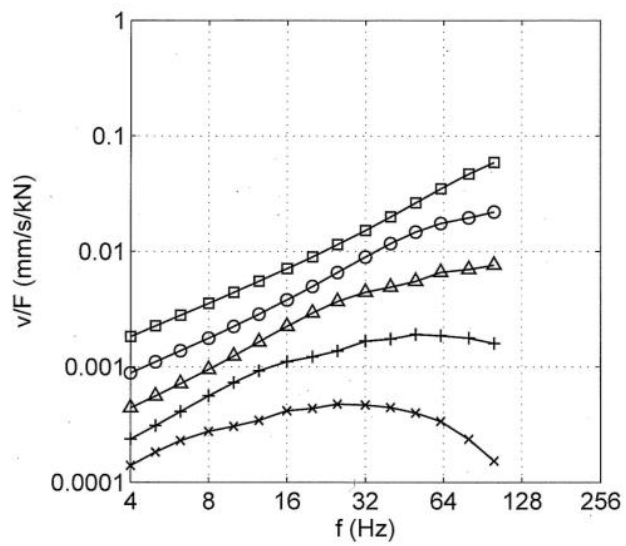

Bild 3: Übertragungsfunktionen einer Schicht $\left(H=8 \mathrm{~m}, v_{S}=150 \mathrm{~m} / \mathrm{s}\right)$ auf einem steiferen Halbraum $\left(v_{s}=300 \mathrm{~m} / \mathrm{s}\right)$ a) an der Oberfläche, b) in $\left.4 \mathrm{~m}, \mathrm{c}\right)$ in $8 \mathrm{~m}$, d) in $12 \mathrm{~m}$ Tiefe, e) Halbraum mit $v_{S}=300 \mathrm{~m} / \mathrm{s}$, f) Vollraum mit $v_{S}=300 \mathrm{~m} / \mathrm{s}, r=\square 4 \mathrm{~m}, \bigcirc 8 \mathrm{~m}, \triangle 16 \mathrm{~m}$, $+32 \mathrm{~m}, \times 64 \mathrm{~m}$

raums. Die tieffrequenten Amplituden nähern sich dem steifen Halbraum an. Dazwischen bei $8 \mathrm{~Hz}$ ist eine leichte Schichtresonanz zu erkennen. Bei einer Tiefenlage von $4 \mathrm{~m}$ (Bild 3b) hat man die abgeschwächten Amplituden des (weichen) Vollraums, zumindest bei den höheren 
a)

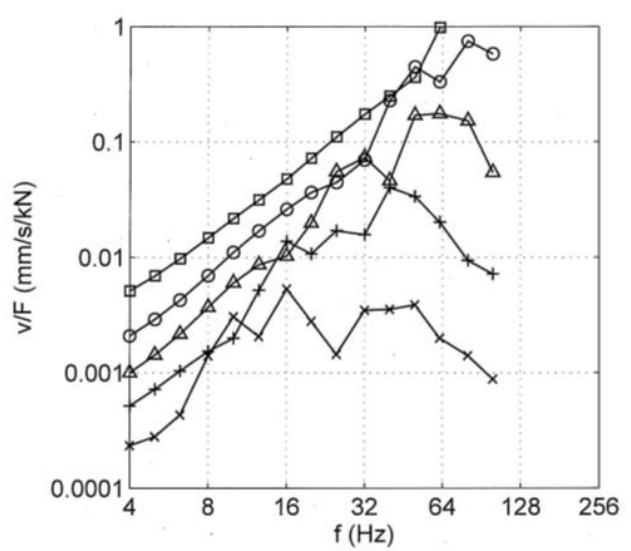

c)

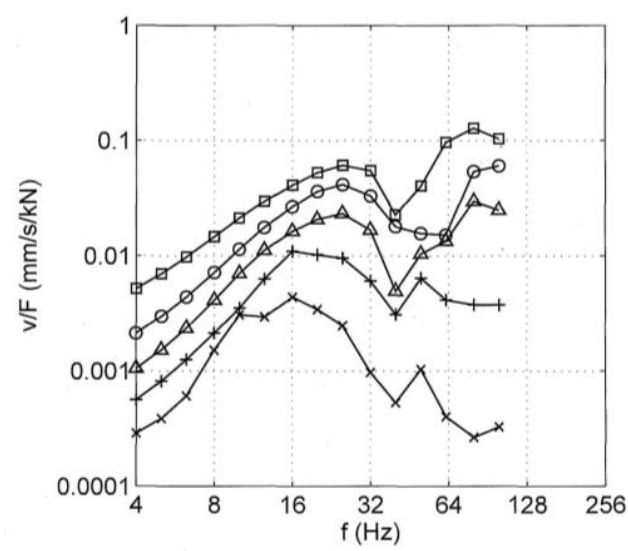

e)

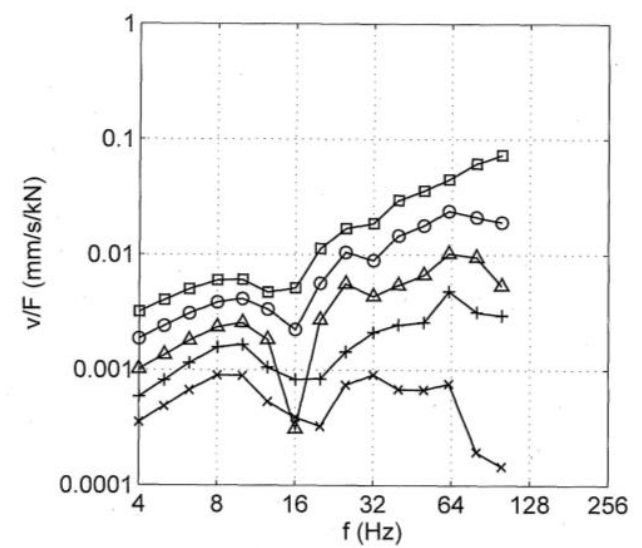

b)

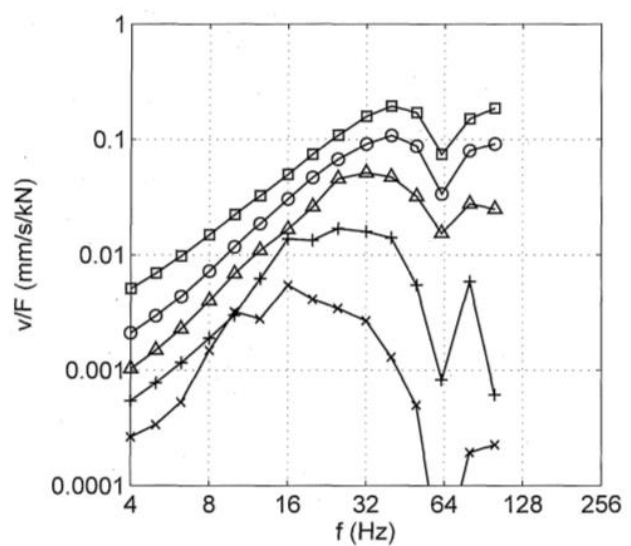

d)

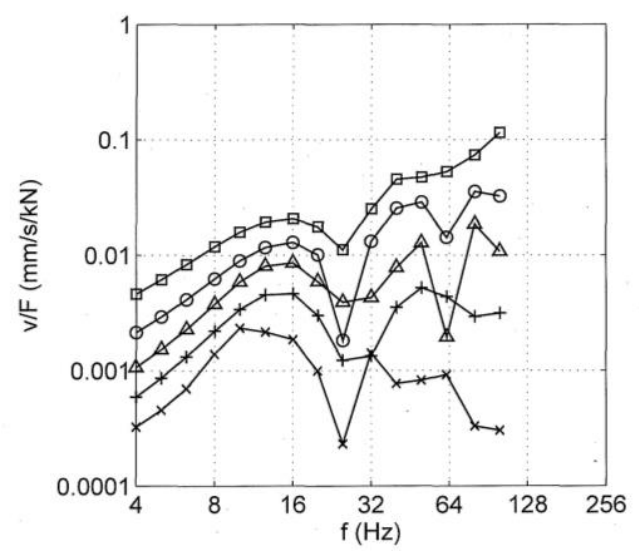

f)

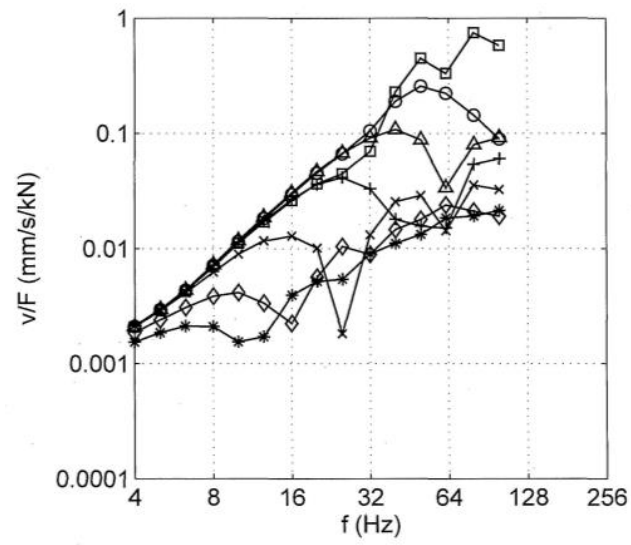

Bild 4: Übertragungsfunktionen eines Bodens mit zunehmender Steifigkeit $\left(v_{s}=100 \ldots\right.$ $315 \mathrm{~m} / \mathrm{s}$ bis $12 \mathrm{~m}$ Tiefe), $r=\square 4 \mathrm{~m}, \bigcirc 8 \mathrm{~m}, \triangle 16 \mathrm{~m},+32 \mathrm{~m}, \times 64 \mathrm{~m}$, a) an der Oberfläche, b) in $1 \mathrm{~m}, \mathrm{c}$ ) in $2 \mathrm{~m}, \mathrm{~d}$ ) in $4 \mathrm{~m}, \mathrm{e}$ ) in $8 \mathrm{~m}, \mathrm{f}$ ) Vergleich für $r=8 \mathrm{~m}, z=\square 0 \mathrm{~m}, \bigcirc 0,5 \mathrm{~m}, \triangle 1 \mathrm{~m}$, $+2 \mathrm{~m}, \times 4 \mathrm{~m}, \diamond 8 \mathrm{~m}, * 12 \mathrm{~m}$

Frequenzen (Bild 2e). Bei einer Tiefenlage von $8 \mathrm{~m}$ (Bild 3c) wirkt die Anregung auf dem steifen Halbraum und die Spektren ähneln tieffrequent denen des steifen Halbraumes (Bild 3e). Bei hohen Frequenzen hat man das Wellenfeld des steifen Vollraums (Bild 3f). Diese Spektren

Zur Prognose von Erschütterungen aus Bahntunneln AUERSCH

2. Wiener Dynamik Tage 2021 
ändern sich kaum, wenn die Tiefenlage bei 12 m liegt (Bild 3d).

In Bild 4 wird ein Boden mit kontinuierlich zunehmender Steifigkeit gemäß $G \sim \sqrt{ } z$ bzw. $v_{S} \sim Z^{0.25}$ untersucht. Dies wird durch ein Bodenmodell mit sieben Schichten angenähert: $v_{S}=$ 100, 140, 170, 200, 240, 285, $315 \mathrm{~m} / \mathrm{s}$ bei $h=0,0,5,1,2,4,8,12 \mathrm{~m}$. An der Bodenoberfläche hat man eine stärkere Amplitudenzunahme, die gleichmäßig vom steifen Halbraum mit $v_{S}=$ $315 \mathrm{~m} / \mathrm{s}$ bis zum weichen Halbraum mit $v_{S}=100 \mathrm{~m} / \mathrm{s}$ führt (Bild 4a). Bei der Tiefenlage der Last erkennt man eine hochfrequente Reduktion, die umso tieffrequenter beginnt (etwa bei $100,64,40,25,16,12 \mathrm{~Hz}$ ), je größer die Tiefenlage ist. Das ist besonders gut in der Zusammenstellung aller Tiefenlagen in Bild $4 \mathrm{f} z u$ verfolgen. Bei hohen Frequenzen werden die Amplituden des jeweils angeregten Vollraumes erreicht.

\section{Das Wellenfeld einer Punkt- oder Zuglast}

Im vorangehenden Abschnitt wurden die Antworten auf Punktlasten beschrieben. Die Antwort auf eine Zuglast erhält man durch die Überlagerung der Antworten auf $n$ über die Zuglänge $L$ verteilten Achslasten. Die Achslasten werden als zufällige unabhängige und ortsfeste Lasten betrachtet, deren Antworten bzw. Übertragungsfunktionen als Wurzelquadratsumme addiert werden

$$
H_{Z}(x, f)=\sqrt{\sum_{j=1}^{n} H_{P}{ }^{2}\left(\sqrt{x^{2}+y_{j}^{2}}, f\right)},
$$

Dabei werden $n=40$ and $L=250 \mathrm{~m}$ als Standardwerte benutzt, die bei normalen Böden mit normaler Dämpfung und bei Entfernungen bis $100 \mathrm{~m}$ schon die Gesetzmäßigkeiten eines
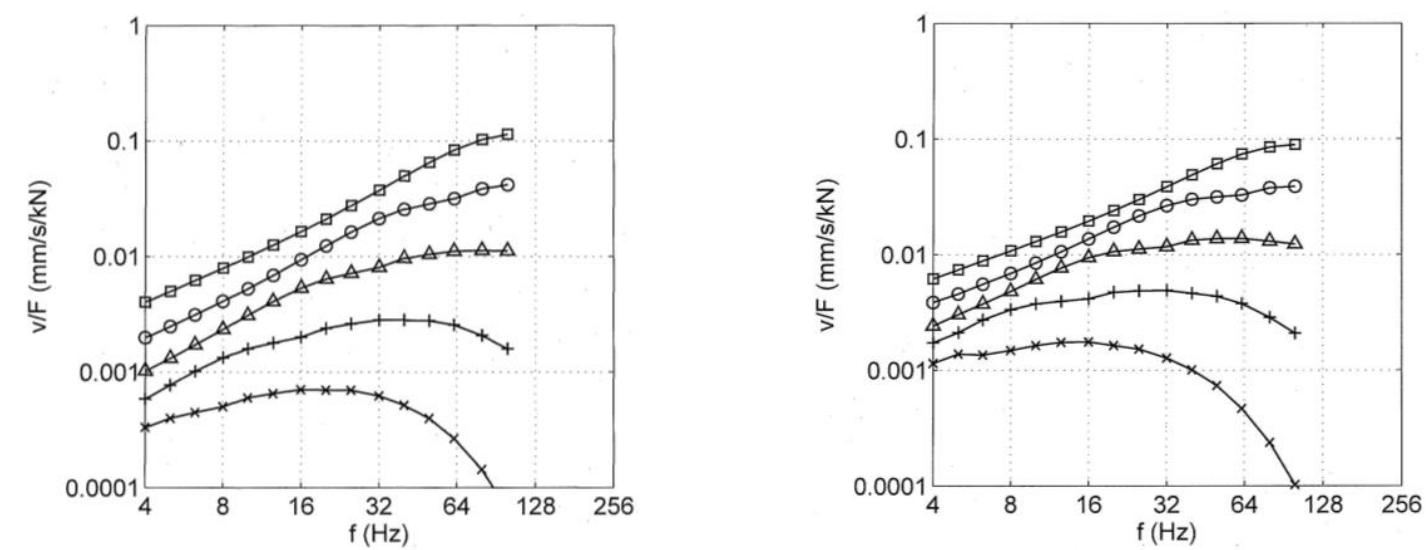

Bild 5: Übertragungsfunktionen eines homogenen Bodens mit $v_{s}=200 \mathrm{~m} / \mathrm{s}, r=\square 4 \mathrm{~m}, \bigcirc 8 \mathrm{~m}$, $\triangle 16 \mathrm{~m},+32 \mathrm{~m}, \times 64 \mathrm{~m}$, a) für eine Punktlast, b) für eine Zuglast

Zur Prognose von Erschütterungen aus Bahntunneln AUERSCH

2. Wiener Dynamik Tage 2021 
unendlich langen Zuges ergeben. Bild 5 zeigt die Punkt- und die Zuglastlösung für einen Vollraum mit $v_{S}=200 \mathrm{~m} / \mathrm{s}$. Bei tiefen Frequenzen ist die Amplitudenabnahme für die Punktlast deutlich stärker als für die Zuglast. Bei hohen Frequenzen dominiert die Wirkung der Materialdämpfung, so dass die Unterschiede zwischen Punkt- und Zuglast weniger auffallen.

\section{Schwingungsverhalten des Tunnels und Auswirkungen auf die Emission}

Es wird das Schwingungsverhalten eines Tunnels mit dem Querschnitt $6 \mathrm{~m} \times 9 \mathrm{~m}$, einer Wandstärke (Beton) von $d=0,7 \mathrm{~m}$ in einem Boden (Vollraum) von $v_{S}=200 \mathrm{~m} / \mathrm{s}$ untersucht.
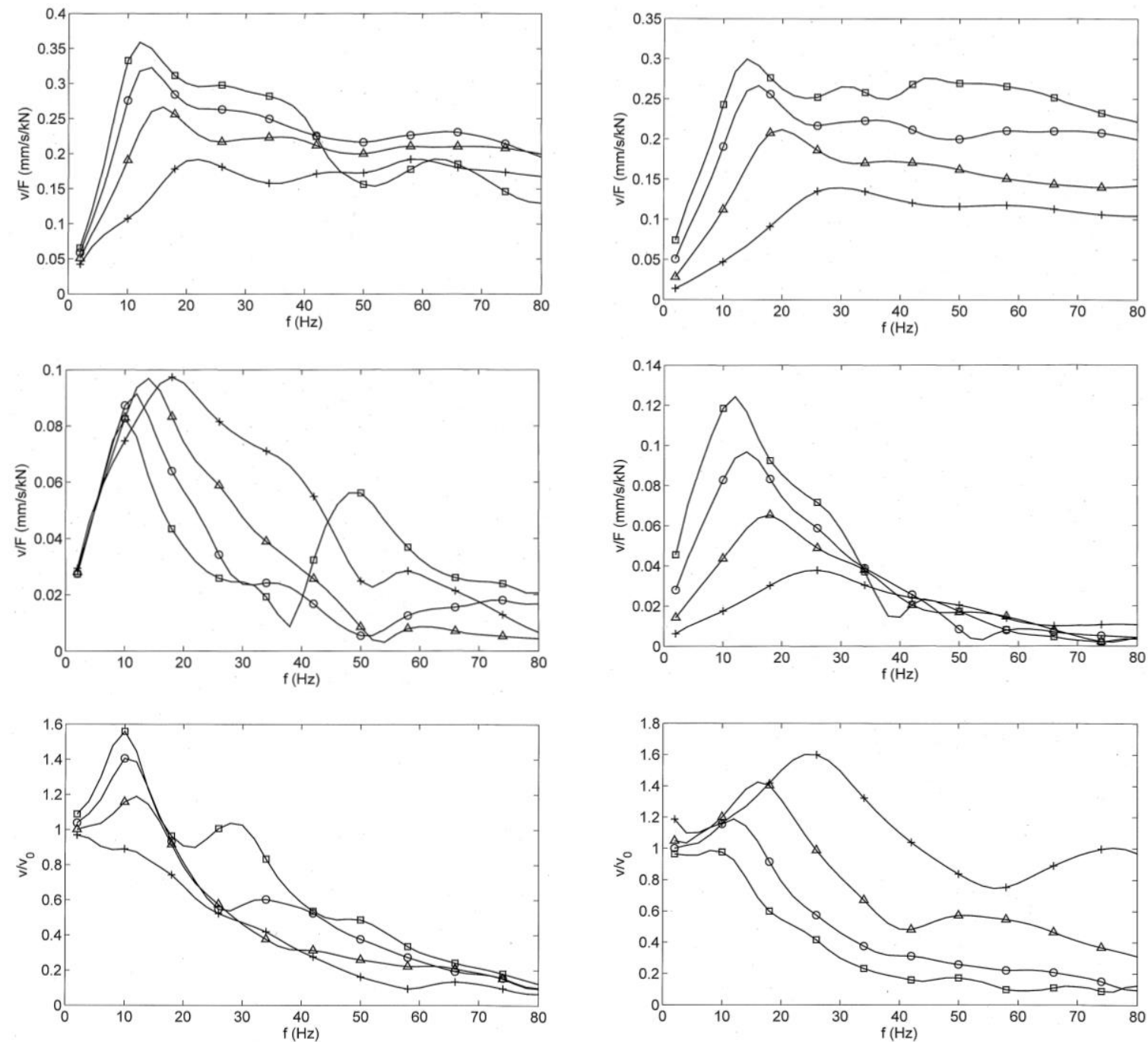

Bild 6: Verhalten des Tunnels a,b) Admittanz der Tunnelsohle, c,d) Admittanz der Tunneldecke, e,f) Minderung der Erschütterungsemission des Tunnels im Vergleich zur Punktlastanregung; a,c,e) Variation der Tunneldicke $\square 0,3 \mathrm{~m}, \bigcirc 0,5 \mathrm{~m}, \triangle \underline{0,7 \mathrm{~m}},+1,0 \mathrm{~m}, \mathrm{~b}, \mathrm{~d}, \mathrm{f})$ Variation des Bodens $\square 150, \bigcirc \underline{200}, \triangle 300,+500 \mathrm{~m} / \mathrm{s}$

Zur Prognose von Erschütterungen aus Bahntunneln AUERSCH

2. Wiener Dynamik Tage 2021 
Die Wandstärke und die Wellengeschwindigkeit des Bodens werden variiert. Bild 6 zeigt als Ergebnis die Admittanz der Tunnelsohle und der Tunneldecke sowie die Erschütterungsminderung der Tunnelemission im Vergleich der Punktlastanregung. Die Sohlenadmittanz nimmt sowohl mit der Wandstärke als auch mit der Wellengeschwindigkeit ab. Die der Deckenadmittanz (Bild 6c) zeigt zumindest in einem mittleren Frequenzbereich einen gegenteiligen Wandstärkeeinfluss. Bei der Erschütterungsminderung ist der Steifigkeitseinfluss vom Tunnel (kleinere Amplituden) und Boden (größere Amplituden) entgegengesetzt. Ein Einfluss mit $v / v_{0} \sim v_{S} / d \omega$ ähnlich wie beim oberirdischen Schienenverkehr scheint als grobe Approximation möglich. Der Einfluss der Wandstärke ist eher kleiner als der Einfluss der Wellengeschwindigkeit. Die Sohlenadmittanz ist annähernd mit der Frequenz konstant, die Deckenadmittanz nimmt deutlich mit der Frequenz ab, und auch die Erschütterungsemission hat ebenfalls eine abnehmende Tendenz mit der Frequenz.

\section{Immision in Gebäuden}

Auch bei der Erschütterungsimmission in Gebäuden gibt es einen Minderungseffekt durch den Lauf der Wellen. Als Beispiel wird eine $12 \mathrm{~m}$ langes Plattenfundament in Ausbreitungsrichtung der Wellen berechnet. Wie zuvor wird die Fundamentdicke und die Wellengeschwindigkeit des Bodens variiert. Bild 7 zeigt das Amplitudenverhältnis des Gebäudefundaments zum Freifeld des Bodens. Es gibt eine mit der Frequenz stärker werdende Minderung. Der Einfluss des Bodens ist stärker als der Einfluss der Fundamentplatte. Allerdings ist die Wirkung eines steifen Bodens gering. Je steifer der Boden desto größer die Amplituden, je steifer die Platte, desto kleiner die Amplituden.
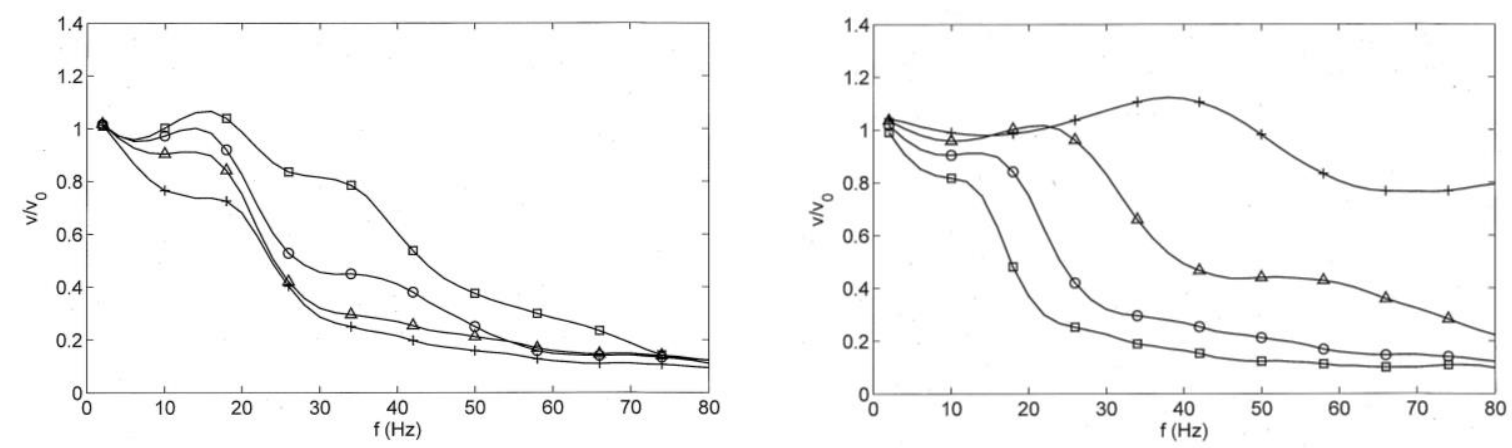

Bild 7: Minderung der Erschütterungsimmission durch die Steifigkeit eines $12 \mathrm{~m}$ langen Gebäudefundaments, a) Variation der Fundamentdicke $\square 0,3 \mathrm{~m}, \bigcirc 0,5 \mathrm{~m}, \triangle \underline{0,7 \mathrm{~m}},+1,0 \mathrm{~m}$, b) Variation des Bodens $\square 150, \bigcirc \underline{200}, \triangle 300,+500 \mathrm{~m} / \mathrm{s}$ 


\section{Zusammenfassung}

Bei der Wellenausbreitung in der Tiefe verliert die Rayleighwelle und ihre Eindringtiefe an Bedeutung. Statt der Halbraumlösung bestimmt die Vollraumlösung stärker das Geschehen. Mit der Vollraumlösung halbieren sich die Amplituden, und bei hohen Frequenzen fällt außerdem die Rayleighwelle weg und es verbleiben Raumwellen als maßgebliche Lösung. Trifft die Anregung in der Tiefe auf steifere Bodenschichten, so ergibt der entsprechende Vollraum die hochfrequente Lösung. Bei tiefen Frequenzen und großen Wellenlängen $(\lambda>z)$ hat man die Oberflächenlösung (die Halbraumlösung des steifen Bodens) und die Tiefenlage macht sich nicht bemerkbar.

Für die Prognose von Erschütterungen aus Bahntunneln braucht man neben den Gesetzmäßigkeiten der Wellenausbreitung für eine Punktlast die Gesetzmäßigkeiten einer Zuglast, die Emissionsminderung durch den steifen Tunnel und die Immisionsminderung durch ein Gebäudefundament. Entsprechende Ergebnisse werden ebenfalls mitgeteilt.

\section{Literaturangaben}

[1] L. Auersch: Simple and fast prediction of train-induced track forces, ground and building vibrations. Railway Engineering Science, 28, 2020, 232-250.

[2] L. Auersch: Wave propagation in the elastic half-space due to an interior load and its application to ground vibration problems and buildings on pile foundations. Soil Dynamics and Earthquake Engineering 30, 2010, 925-936.

[3] L. Auersch: Wave propagation in layered soil: theoretical solution in wavenumber domain and experimental results of hammer and railway traffic excitation. Journal of Sound and Vibration 173, 1994, 233-264.

[4] L. Auersch, G. Schmid: A simple boundary element formulation and its application to wavefield excited soil-structure interaction. Earthquake Engineering and Structural Dynamics 19, 1990, 931-947.

Zur Prognose von Erschütterungen aus Bahntunneln AUERSCH

2. Wiener Dynamik Tage 2021 